\title{
A NOTE ON ORDER COMPLETE $f$-ALGEBRAS
}

\author{
BORIS LAVRIČ
}

\begin{abstract}
Let $A$ be an Archimedean uniformly complete $f$-algebra with unit element. Then the following conditions are equivalent

(i) $A$ is order complete.

(ii) Every regular algebra ideal in $A$ is an order ideal. ideal.

(iii) Every finitely generated regular algebra ideal in $A$ is a principal algebra

The proof is based on the fact that the range of every injective orthomorphism in an order complete Riesz space is an order ideal.
\end{abstract}

1. Introduction. Let $L$ be an Archimedean Riesz space. The sequence $\left(f_{n}\right.$ : $n \in \mathbf{N})$ in $L$ is called an order Cauchy sequence whenever there exists a sequence $\left(p_{n}: n \in \mathbf{N}\right)$ in $L$ such that $p_{n} \downarrow 0$ and $\left|f_{n}-f_{n+k}\right| \leq p_{n}$ for all $k, n \in \mathbf{N}$. The Riesz space $L$ is said to be order complete if every order Cauchy sequence in $L$ is order convergent. The following criterion for order completeness due to Papangelou [7] is well known. The Riesz space $L$ is order complete if and only if for any pair of sequences $\left(f_{n}: n \in \mathbf{N}\right),\left(g_{n}: n \in \mathbf{N}\right)$ in $L$ satisfying $f_{n} \uparrow \leq g_{n} \downarrow$ and $\inf \left(g_{n}-f_{n}\right)=0$ there exists an element $h \in L$ such that $f_{n} \leq h \leq g_{n}$ for all $n \in \mathbf{N}$.

An order complete Riesz space is uniformly complete and the family of all order complete Riesz spaces contains all uniformly complete normal Riesz spaces. It is shown in [4, Corollary 5.3] that the range of every orthomorphism (i.e. order bounded band preserving linear map) in a uniformly complete normal Riesz space is an order ideal. What can be said if we consider only injective orthomorphisms? In the first part of this note it is shown that the order complete Riesz spaces can substitute the normal ones in the above conclusion.

This result is used to give some characterizations of order completeness of uniformly complete Archimedean $f$-algebras with unit. They are presented in Theorem 4, which generalizes [2, Theorem 5.1] concerning algebras $C(X)$ of all real valued continuous functions on a completely regular Hausdorff space $X$. Examples show that the condition of uniform completeness cannot be dropped in Theorem 4 .

For terminology and elementary properties of Riesz spaces and $f$-algebra theory not explained here we refer the reader to $[\mathbf{5 , 8 , 4}]$.

Finally we want to emphasize that all proofs in this note will be free of any representation, so that Zorn's lemma is not used unnecessarily.

2. The range of an injective orthomorphism. We shall begin with a property characteristic for an injective orthomorphism.

Received by the editors December 5, 1985 and, in revised form, April 16, 1986.

1980 Mathematics Subject Classification (1985 Revision). Primary 06A65, 06A70; Secondary 47 B55.

Key words and phrases. Riesz space, $f$-algebra, orthomorphism.

This work was supported in part by the Research Council of Slovenia, Yugoslavia. 
Proposition 1. Let $L$ be an Archimedean Riesz space, and let $T: L \rightarrow L$ be an order bounded linear operator. Then the following two condition are equivalent.

(i) $T$ is an injective orthomorphism.

(ii) $\{T u\}^{d d}=\{u\}^{d d}$ for all $u \in L$.

ProOF. (i) implies (ii): Since $T$ is band preserving, $\{T u\}^{d d} \subset\{u\}^{d d}$ holds for all $u \in L$. For the converse inclusion we have to show that $u \in\{T u\}^{d d}$. To this end take $v \in\{T u\}^{d}$; note that $|v| \wedge|T||u|=|v| \wedge|T u|=0$. By virtue of $|T|(|v| \wedge|u|)=|T||v| \wedge|T||u|=0,|v| \wedge|u|=0$, since $T$ is injective.

(ii) implies (i): Trivial.

Let us continue with a result on the range of an injective orthomorphism.

THEOREM 2. Let $L$ be an Archimedean order complete Riesz space. Then the range $R(T)$ of an injective orthomorphism $T: L \rightarrow L$ is an order ideal.

ProOF. Assume first that $T$ is a positive and injective orthomorphism on $L$ and take an arbitrary $u \in L^{+}$. It is enough to see that every $0 \leq v \leq T u$ lies in the range $R(T)$ of $T$. For every $n \in \mathbf{N}$ set

$$
p_{n}=(T \vee(1 / n) I)^{-1} v, \quad q_{n}=u-(T \vee(1 / n) I)^{-1}(T u-v),
$$

where $I$ denotes the identity of $L$. We will prove that $p_{n} \uparrow \leq q_{n} \downarrow$ and $\left(q_{n}-p_{n}\right) \downarrow 0$. It is obious that $p_{n} \uparrow$ and $q_{n} \downarrow$. Since $u \in\{T u\}^{d d}$ by Proposition 1 , the relation $(u \wedge n \cdot T u) \uparrow u$ holds. Therefore

$$
\begin{aligned}
q_{n}-p_{n} & =u-(T \vee(1 / n) I)^{-1} T u=u-(I \wedge n \cdot T) u \\
& =(u-(u \wedge n \cdot T u)) \downarrow 0
\end{aligned}
$$

As $L$ is order complete, there exists an element $h \in L$ such that $p_{n} \leq h \leq q_{n}$ for all $n \in \mathbf{N}$, so $p_{n} \uparrow h, q_{n} \downarrow h$, and $T p_{n} \uparrow T h, T q_{n} \downarrow T h$, since $T$ is order continuous. On the other hand

$$
T p_{n}=T(T \vee(1 / n) I)^{-1} v=(I \wedge n T) v=(v \wedge n T v) \uparrow v
$$

hence $v=T h \in R(T)$ as we claimed.

Now let $T$ be an arbitrary injective orthomorphism on $L$. Then $|T|$ is an injective orthomorphism with the range $R(|T|)$ an order ideal in $L$. By [3, Lemma 2.7] $R(T)$ is also an order ideal in $L$, and the proof is finished.

Note. For the last part of the proof (i.e., general case of injective orthomorphism) we can proceed also along the lines of [4, Corollary 5.3] or [8, Theorem 146.7].

3. Order complete $f$-algebras. Let $A$ be an Archimedean $f$-algebra with unit element. Recall that by an $r$-ideal $I$ in $A$ we mean an algebra ideal $I \subset A$, that the $r$-ideal generated by $a_{1}, \ldots, a_{n} \in A$ is denoted by $\left(a_{1}, \ldots, \dot{a}_{n}\right)$, and an $r$-ideal which is in addition an order ideal is called an $l$-ideal.

We shall say that an element $a \in A$ is regular if $a b=0, b \in A$ implies $b=0$. An $r$-ideal $J \subset A$ is said to be regular if it contains a regular element. Since a square of a regular element is regular, every regular $r$-ideal contains a positive regular element. Obviously $a \in A$ is regular if and only if $|a|$ is a weak unit in $A$.

In [2, Theorem 5.1] it is proved that for the algebra $C(X)\left(\right.$ or $\left.C_{b}(X)\right)$ of all real continuous (and bounded) functions on a completely regular Hausdorff topological 
space $X$ the following statements are equivalent: $X$.

(a) $X$ is a quasi- $F$ space, i.e., every dense cozero set $C \subset X$ is $C_{b}$-embedded in

(b) $C(X)$ is an order complete Riesz space.

(c) Every regular $r$-ideal in $C(X)$ is an $l$-ideal.

(d) Every finitely generated regular $r$-ideal in $C(X)$ is a principal $r$-ideal.

We shall examine the conditions (b), (c), and (d) in a more general context of Archimedean unital $f$-algebras.

First we present some conditions which are equivalent to condition (c).

Proposition 3. Let $A$ be an Archimedean $f$-algebra with unit element $e$. Then the following statements are equivalent.

(i) For every weak unit $w \in A^{+}$the principal $r$-ideal $(w)$ is an l-ideal.

(ii) For every regular element $a \in A$ the principal $r$-ideal $(a)$ is an l-ideal.

(iii) Every regular $r$-ideal in $A$ is an l-ideal.

(iv) $(a, b)=(a+b)$ for any $a, b \in A^{+}$with $a+b$ regular.

ProOF. (i) implies (ii): Take a regular $a \in A$, note that $|a|$ is a weak unit and by (i) $(|a|)$ is an $l$-ideal. Thus $a^{+}, a^{-} \in(|a|), a=k|a|$ for some $k \in A$; therefore $|a|=k a$ and so $(a)=(|a|)$ is an $l$-ideal.

(ii) implies (iii): Let $J \subset A$ be a regular $r$-ideal in $A$ and $w \geq 0$ a regular element in $J$. Observe first that for $0 \leq a \in J$ and $0 \leq b \leq a$ we have $b=f(a+w)$ for some $f \in A$, since $(a+w)$ is an $l$-ideal by (ii), so $b \in J$.

Now, let $a \in J$ be arbitrary. We will show that $|a| \in J$, and (iii) will follow. Set $u=\left(w-a^{-}\right)^{+} \leq w$ and note that $u=g w$ for some $0 \leq g \leq e, u a^{+}=$ $w a^{+}$, so $w a^{+}=g w a^{+}$; therefore $a^{+}=g a^{+}$. Next observe that from the obvious inequality $\left(w-a^{-}\right)^{2} \geq-w a^{-}$it follows that $w^{2} \geq\left(w-a^{-}\right) a^{-}$, consequently $w^{2} \geq\left(w-a^{-}\right)^{+} a^{-}=u a^{-}=g w a^{-}$and $w \geq g a^{-} \geq 0$. Now put $f=2 g-e$ and see that $|a|-f a=2\left(a^{+}-g a\right)=2 g a^{-} \in[0,2 w] \subset J$. Hence $|a| \in J$, as we claimed.

(iii) implies (iv): Let $a, b \in A^{+}$with $a+b$ a weak unit in $A$. By (iii) $(a+b)$ is an $l$-ideal, so $a, b \in(a+b)$ and (iv) follows immediately.

(iv) implies (i): Let $w$ be a weak unit in $A, a \in(w)$, and $|b| \leq|a|$. Then $a=k w$ for some $k \in A, 0 \leq b^{ \pm} \leq|k| w$, and $\left(b^{ \pm},(|k|+e) w-b^{ \pm}\right)=((|k|+e) w) \subset(w)$, since (iv) holds. Therefore $b \in(w)$ and the proof is complete.

In the next theorem we compare the conditions (b), (c), and (d).

THEOREM 4. Consider the following conditions for the Archimedean f-algebra $A$ with unit element $e$.

(i) $A$ is order complete.

(ii) Every regular $r$-ideal in $A$ is an l-ideal.

(iii) Every finitely generated regular $r$-ideal in $A$ is a principal $r$-ideal.

Then (i) implies (ii), and (ii) implies (iii). If $A$ is uniformly complete, then the conditions are equivalent.

Proof. (i) implies (ii): Let $w \in A$ be a weak unit in $A$, and $\pi_{w}: A \rightarrow A$ be the orthomorphism defined by $\pi_{w} a=w a, a \in A$. Since $\pi_{w}$ is injective, the range $(w)$ of $\pi_{w}$ is an $l$-ideal by Theorem 2, and (ii) holds by Proposition 3 .

(ii) implies (iii): Let $J=\left(a_{1}, \ldots, a_{n}\right)$ be a regular $r$-ideal generated by $a_{1}, \ldots, a_{n}$ $\in A$. Then it is easy to see that $w=\left|a_{1}\right|+\cdots+\left|a_{n}\right|$ is a weak unit in $A$ and $J \subset(w)$, 
since $J$ and $(w)$ are $l$-ideals. On the other hand $\left|a_{i}\right| \in J, i=1, \ldots, n$, therefore $(w) \subset J$. Both inclusions together give the equality $(w)=J$.

Assume now that $A$ is in addition uniformly complete. We will show that (iii) implies (i) and the proof of the theorem will be finished. Let $a, b \in A^{+}$with $a+b$ a weak unit in $A$. The $r$-ideal $(a, b)$ is by assumption a principal $r$-ideal, say equal to $(w)$. Hence $a=u|w|$ and $b=v|w|$ for some $u, v \in A^{+}$. Since there exist elements $f, g \in A$ such that

$$
w=a f+b g=u|w| f+v|w| g=(u f+v g)|w|,
$$

we get $|u f+v g|=e$, so $(u+v)^{-1}$ exists in $A$ ( $A$ is uniformly complete!). It follows that

$$
a=u|w|=(u+v) \cdot(u+v)^{-1} u|w|=(u+v)^{-1} u(a+b)
$$

and similarly we have $b=(u+v)^{-1} v(a+b)$. Since $a+b$ is regular, this gives the relations $u \in\{a\}^{d d}$ and $v \in\{b\}^{d d}$, hence

$$
e=(u+v)^{-1} u+(u+v)^{-1} v \in\{a\}^{d d}+\{b\}^{d d} .
$$

Then obviously $A=\{a\}^{d d}+\{b\}^{d d}$ and by [6, Theorem 11.7] $A$ is order complete.

If $A$ is an Archimedean unital $f$-algebra, the injective orthomorphisms on $A$ are precisely multiplications with regualr elements of $A$, i.e., $\pi \in \operatorname{Orth}(A)$ and $\pi$ is injective if and only if $\pi=\pi_{a}: A \rightarrow A ; \pi_{a} f=a f, f \in A$ with $a \in A$ regular. So we conclude from the above theorem and Proposition 3 that the range of an injective orthomorphism on a uniformly complete unitary $f$-algebra $A$ is an order ideal if and only if $A$ is order complete. Hence we cannot expect that Theorem 2 holds under weaker conditions.

The following two examples will show that the reverse implication (ii) $\Rightarrow$ (i) and (iii) $\Rightarrow$ (ii) do not hold in a nonuniformly complete $f$-algebra.

EXAMPLE 5. Let $A$ be the Archimedean unital $f$-algebra of all real functions with finite range defined on $[0,1] \subset \mathbf{R}$. Every regular element of $A$ is invertible, therefore $(w)=A$ for all weak units $w \in A$, hence (ii) of Theorem 4 holds. But (i) does not hold, since $A$ is not uniformly complete.

EXAMPLE 6 (SEE [4, EXAMPLE 6.7]). Let $A$ be the $f$-algebra of all real sequences $a=\left(a_{1}, a_{2}, \ldots\right)$ satisfying the following condition. For every $a \in A$ there exists $n_{a} \in \mathbf{N}$ and a polynomial $p_{a} \in \mathbf{R}[X]$ such that $a_{n}=p_{a}(n)$ for all $n \geq n_{a}$. It can be seen that every finitely generated $r$-ideal in $A$ is principal, so (iii) holds. On the other hand the $r$-ideal $(w)$ generated by the weak unit $w \in A, w_{n}=n+1, n \in \mathbf{N}$, is not an $l$-ideal. Indeed, $a \in A, a_{n}=n, n \in \mathbf{N}$, satisfies $0 \leq a \leq w$, but $a \notin(w)$.

Observe that $A$ satisfies (iii), but the range $(w)$ of the orthomorphism $\pi_{w}: A \rightarrow$ $A, \pi_{w} f=w f, f \in A$, is not an order ideal.

REMARKS. Theorem 4 can be compared with [4, Theorem 6.6], which says in particular that for an Archimedean unital uniformly complete $f$-algebra $A$ the following conditions are equivalent: (i) $A$ is normal; (ii) every $r$-ideal in $A$ is an $l$ ideal; and (iii) every finitely generated $r$-ideal in $A$ is a principal $r$-ideal. Considering this analogy, and recalling that an Archimedean unital $f$-algebra $A$ is normal if and only if $(a)=(|a|)$ for every $a \in A$ [4, Proposition 6.3], a natural question arises. Is $A$ order complete if and only if the condition $(a)=(|a|)$ holds for every regular element $a \in A$ ? An order complete unital $f$-algebra satisfies the above condition, but [2, Example 5.6] shows that the converse fails in general. 
As observed by the referee, Theroems 3 and 4 can be obtained also using $[1,2.3$ and 2.8] in combination with [2, Theorem 5.1], of course, applying the representation theory.

ADDED IN PROOF. After this paper was submitted, we were informed that our Theorem 2 and Theorem 4 have been proved by B. de Pagter in his doctoral thesis f-algebras and orthomorphisms (Leiden, 1981).

\section{REFERENCES}

1. E. R. Aron and A. W. Hager, Convex vector lattices and l-algebras, Topology Appl. 12 (1981), $1-10$.

2. F. K. Dashiell, Jr., A. W. Hager, and M. Henriksen, Order Cauchy completions of rings and vector lattices of continuous functions, Canad. J. Math 32 (1980), 657-685.

3. D. R. Hart, Some properties of disjointness preserving operators, Indag. Math. 47 (1985), 183197.

4. C. B. Huijsmans and B. de Pagter, Ideal theory in f-algebras, Trans. Amer. Math. Soc. 269 (1982), 225-245.

5. W. A. J. Luxemburg and A. C. Zaanen, Riesz spaces. I, North-Holland, Amsterdam and London, 1971.

6. B. de Pagter, On z-ideals and d-ideals in Riesz spaces. III, Indag. Math. 43 (1981), 409-422.

7. F. Papangelou, Order convergence and topological completion of commutative lattice groups, Math. Ann. 155 (1964), 81-107.

8. A. C. Zaanen, Riesz spaces. II, North-Holland, Amsterdam, 1983.

Department of Mathematics, E. K. University of Ljubljana, Ljubljana, YuGOSLAVIA 